\title{
Narrow linewidth intervalence-band emission from germanium THz lasers
}

\author{
D. R. Chamberlin ${ }^{\mathrm{a}}$ \\ Agilent Laboratories, Palo Alto, CA 94304 \\ E. Bründermann \\ Ruhr-Universität Bochum, D-44780 Bochum, Germany \\ E. E. Haller \\ Lawrence Berkeley National Laboratory, Berkeley, CA 94720 and \\ University of California, Berkeley, CA 94720
}

\begin{abstract}
We demonstrate narrow linewidth emission from $\mathrm{p}-\mathrm{Ge} \mathrm{THz}$ lasers at low magnetic fields where intervalence band emission dominates. The volume of these lasers crystals is $32 \mathrm{~mm}^{3}$, which is one order of magnitude smaller than the lasers for which the emission spectrum has been measured previously. The frequencies emitted are shown to be selected by the optical cavity and do not shift with the magnetic field as has been observed for larger laser crystals.
\end{abstract}

42.55.Px, 42.60.Fc, 42.60.By, 42.72.Ai

a Email: danielle_chamberlin@agilent.com 
A number of important applications exist for a compact, tunable, continuous-wave terahertz (THz) laser. For instance, astronomers need such a coherent source for highresolution heterodyne spectroscopy of star-forming regions to determine chemical constituents, their distribution, and temperature redistribution. ${ }^{1}$ Recent results also show promise for $\mathrm{THz}$ medical and dental imaging. ${ }^{2}$ A very promising $\mathrm{THz}$ source for such applications is the $\mathrm{p}$-Ge laser operating with a hole population inversion. While much recent work has focused on generation of ultra-short pulses by mode-locking the Ge laser, ${ }^{3,4,5}$ our focus is to reach $\mathrm{CW}$ operation. To accomplish this, the ratio of input power to cooling surface must be reduced. This can be achieved by shrinking the crystal size. ${ }^{6,7}$ Previously we have reported high duty cycle and continuous terahertz emission from small Ge laser crystals. ${ }^{8}$ Recent studies ${ }^{8,9}$ have demonstrated operation of $p$-Ge lasers with volumes as small as $0.5 \mathrm{~mm}^{3}$, however, the spectral output of small laser crystals has not been measured previously due to their low output power of a few tens of milliwatts. In this study we use a Michelson interferometer with a stepping mirror movement and long collecting times at each mirror position to measure the spectral output of these small germanium lasers.

Ge single crystals were grown from a melt doped with the double acceptor beryllium in a vacuum atmosphere using the Czochralski technique. The resulting laser crystals have Be concentrations of $\sim 10^{14} \mathrm{~cm}^{-3}$. Details of the method of Ge crystal characterization, laser fabrication, and laser testing have been presented elsewhere. ${ }^{6,8,10}$ The magnetic field was applied with a superconducting magnet. No external resonator cavity was used 
because the high refractive index of Ge allows operation using only the polished crystal surfaces as mirrors for internal reflections.

The laser emission was measured in the direction parallel to the magnetic field. The radiation was coupled into a Michelson interferometer with a stainless steel lightpipe. The lasers were operated with a repetition rate of $100 \mathrm{~Hz}$ and a pulse length of $7.5 \mu \mathrm{s}$. The light was measured with a broadband $4.2 \mathrm{~K}$ bolometer. In order to achieve a good signal to noise ratio with the small laser power 400 to 800 laser pulses were averaged at each mirror position and then the signal was boxcar integrated. Because of the slow time response of the bolometer, it was possible to exclude the transients caused by the rapid turn-off of the laser excitation current. This allows for further noise reduction because the crosstalk between the pulse generator and the bolometer is not included in the integrated signal.

Figure 1(a) shows the emission of the large $4 \times 4 \times 20 \mathrm{~mm}^{3}$ laser from Reichertz et al. ${ }^{11}$ The electrical contacts were placed on the $4 \times 20 \mathrm{~mm}^{2}$ sides perpendicular to the [001] direction, and the magnetic field was applied parallel to a [110] direction. The light was measured parallel to the long axis of the laser and parallel to a [110] direction. This laser exhibits broadband intervalence-type emission with a FWHM of about $10 \mathrm{~cm}^{-1}$. The emission is tunable over the entire range of 40 to $130 \mathrm{~cm}^{-1}$ with no gaps.

In contrast, figure 1(b) shows the emission of a 10 times smaller laser with dimensions $3.8 \times 3.8 \times 2 \mathrm{~mm}^{3}$. In this laser the electrical contacts were placed on the $3.8 \times 3.8 \mathrm{~mm}^{2}$ sides, and the magnetic field was applied parallel to one of the $3.8 \mathrm{~mm}$ directions of the 
laser. The crystallographic orientations of the electric and magnetic fields were identical to those applied to the larger laser. The smaller laser operates only between $0.6 \mathrm{~T}$ and 1.0 $\mathrm{T}$ because of the smaller gain volume. In addition, it shows sharp lines with FWHM $\leq 1$ $\mathrm{cm}^{-1}$ that do not shift smoothly with magnetic field. Rather, as the magnetic field is increased new discreet emission lines appear at higher energy and the lower-energy lines disappear.

Figure 2(a) shows the laser emission of another $3.8 \times 3.8 \times 2 \mathrm{~mm}^{3}$ laser that was fabricated from the same wafer as the laser shown in figure 1(b). It also emits in discrete lines which do not tune smoothly with the magnetic field, however, the emission lines differ from those observed in figure 1(b). Since the material is exactly the same in both lasers, this suggests that the laser emission is highly dependent on the exact details of the bare germanium surfaces used as the resonator cavity.

To test this hypothesis, this laser was etched in a mixture of 20:1 $\mathrm{HNO}_{3}: \mathrm{HF}$ for 90 seconds. This removed $22 \mu \mathrm{m}$ and $13 \mu \mathrm{m}$ from the two $3.8 \mathrm{~mm}$ directions of the laser crystal, while preserving the high quality of laser surface. The height of the laser crystal in the $2 \mathrm{~mm}$ direction was unaffected because the Au electrical contacts mask the germanium from the etch mixture.

The emission of the laser after the etch procedure is shown in figure 2(b). The weak peak at $99.6 \mathrm{~cm}^{-1}$ has disappeared and has been replaced by a peak at $104.5 \mathrm{~cm}^{-1}$. However, the peak at $78.1 \mathrm{~cm}^{-1}$ is still present. At $0.6 \mathrm{~T}$, an additional peak appears at $84.0 \mathrm{~cm}^{-1}$. This increase in the number of emission peaks can be attributed to the increase in the 
quality of the optical cavity after etching, which was also indicated by an increase in the outer range of $\mathrm{E}$ and $\mathrm{B}$ fields over which the laser operated (figure 3). At $0.8 \mathrm{~T}$ and 1.16 $\mathrm{kV} / \mathrm{cm}$, no emission is observed after etching, but if the electric field is lowered slightly to $0.91 \mathrm{kV} / \mathrm{cm}$ the $104.5 \mathrm{~cm}^{-1}$ peak appears - clearly the intervalence band transitions with enough gain to lase have been significantly changed by the perturbation in the optical cavity.

Although the emissions shown in Figures 1(b) and 2 show very narrow peaks, they are spectrally not fully resolved. In order to determine the peak width of the emission from these near-threshold lasers, one measurement was taken of the laser shown in figure 2 at $0.6 \mathrm{~T}$ and $0.8 \mathrm{kV} / \mathrm{cm}$ with a resolution of $0.03 \mathrm{~cm}^{-1}$. The laser was pulsed at $100 \mathrm{~Hz}$ and 20 averages were taken at each point. There were emission peaks at 78.3 and $84.5 \mathrm{~cm}^{-1}$. Due to the high resolution of the data, it is necessary to display only small portions of the data surrounding the two peaks in order to visualize the peak widths (Figure 4(a) and (b), respectively). Even at this high resolution, it is evident that the spectrum is not yet fully resolved. The peaks show a total width of about $0.1 \mathrm{~cm}^{-1}$ with some more narrow structures within the peak. It is possible that the emission is from single optical modes and the structure in the center of the peak is due to the structure of the closely spaced, final heavy hole states of the transition. Another possibility is that the structure observed originates in the interplay of several optical modes, since there are multiple mode geometries allowed by total internal reflection on the crystal faces. It is impossible to determine conclusively where the minima of the optical cavity transmission lie because an accurate measurement of the optical cavity is not possible due to curvature of the etched germanium surfaces. Recently Muravjov et al demonstrated operation of a large 
p-Ge laser crystal in a tunable external cavity. ${ }^{12}$ Use of such an external cavity with these small laser crystals would separate out the effects of the optical cavity and the gain spectrum.

In summary, the emission spectrum of small crystals near the lasing threshold has been investigated and has shown significant departures from the emission previously observed for more powerful lasers. In contrast to the broadband, tunable emission seen from larger laser crystals, the small lasers emit in specific, narrow frequency bands that do not tune smoothly with magnetic field. This emission is highly dependent on the specific optical cavity. High-resolution spectra show a narrow linewidth of about $0.1 \mathrm{~cm}^{-1}$ with an even narrower substructure suggesting a modal linewidth in the $\mathrm{MHz}$ range. The current results together with previously demonstrated small laser volumes ${ }^{8}$ in this homogeneous laser medium suggest that a small continuous-wave $\mathrm{THz}$ laser would naturally operate in a single mode having mode linewidths on the order of MHz. ${ }^{13}$ Planar and thin layer Ge lasers with small active volumes will allow integration of high $\mathrm{Q}$ tunable resonators on a rather macroscopic scale of millimeters. ${ }^{14}$

This work was supported by the Director, Office of Science, Office of Basic Energy Sciences, Division of Materials Sciences and Engineering, of the U.S. Department of Energy under Contract No. DE-AC03-76SF00098. D. R. C. acknowledges the NASA Office of Space Science for their support. 
${ }^{1}$ R. T. Boreiko and A. L. Betz, Astrophys. J. Suppl. 111, 409 (1997).

2 C. M. Ciesla, D. D. Arnone, A. Corchia, D. Crawley, C. Longbottom, E. J. Linfield, and M. Pepper, Proc. SPIE 3934, 73 (2000).

3 A. V. Muravjov, S. H. Withers, H. Weidner, W. Trimble, S. G. Pavlov, V. N. Shastin, and R. E. Peale, Appl. Phys. Lett. 73, 3037 (1998).

4 J. N. Hovenier, A. V. Muravjov, S. G. Pavlov, V. N. Shastin, R. C. Strijbos, and W. Th. Wenckebach, Appl. Phys. Lett. 71, 443 (1997).

5 J. N. Hovenier, R. M. de Kleijn, T. O. Klassen, W. Th. Wenckebach, D. R. Chamberlin, E. Bründermann, and E. E. Haller, Appl. Phys. Lett. 77, 3155 (2000).

${ }^{6}$ E. Bründermann, A. M. Linhart, L. Reichertz, H. P. Röser, O. D. Dubon, W. L. Hansen, G. Sirmain, and E. E. Haller, Appl. Phys. Lett. 68, 3075 (1996).

${ }^{7}$ P. D. Coleman and D. W. Cronin, Int. J. Infrared and Millimeter Waves 18, 1241 (1997).

8 E. Bründermann, D. R. Chamberlin, and E. E. Haller, Appl. Phys. Lett. 76, 2991 (2000).

9 E. Bründermann, D. R. Chamberlin, and E. E. Haller, Appl. Phys. Lett. 73, 2757 (1998).

${ }^{10}$ E. E. Haller and E. Bründermann, US Patent No. 6,011,810 (4 January 2000).

${ }^{11}$ L. A. Reichertz, O. D. Dubon, G. Sirmain, E. Bründermann, W. L. Hansen, D. R. Chamberlin, A. M. Linhart, H. P. Röser, and E. E. Haller, Phys. Rev. B 56, 12069 (1997). 
12 A. V. Muravjov, E. W. Nelson, R. E. Peale, V. N. Shastin, and C. J. Fredricksen, Infrared Phys. Tech. 44, 75 (2003).

13 E. Bründermann, H. P. Röser, A. V. Muravjov, S. G. Pavlov, and V. N. Shastin, Infrared. Phys. Technol. 36, 59 (1995).

14 D. R. Chamberlin, E. Bründermann, and E. E. Haller, Appl. Phys. Lett. 74, 3761 (1999). 


\section{Figure Captions}

Figure 1: (a) Spectral emission of Ge:Be laser with dimensions 4x4x20 mm3. (b) Spectral emission of Ge:Be laser with dimensions 3.8x3.8x2 mm3.

Figure 2:(a) Emission of a 3.8x3.8x2 $\mathrm{mm} 3$ laser as fabricated. A different laser crystal is used than the one shown in figure 1(b). (b) Emission of same laser after a short etch.

Figure 3: Area in parameter space in which the laser of figure 2 operates. Dotted line: before etch, solid line: after etch.

Figure 4: High-resolution spectrum of the laser in figure 2 at $0.8 \mathrm{kV} / \mathrm{cm}$ and $0.6 \mathrm{~T}$. Resolution is $0.03 \mathrm{~cm}-1$. 

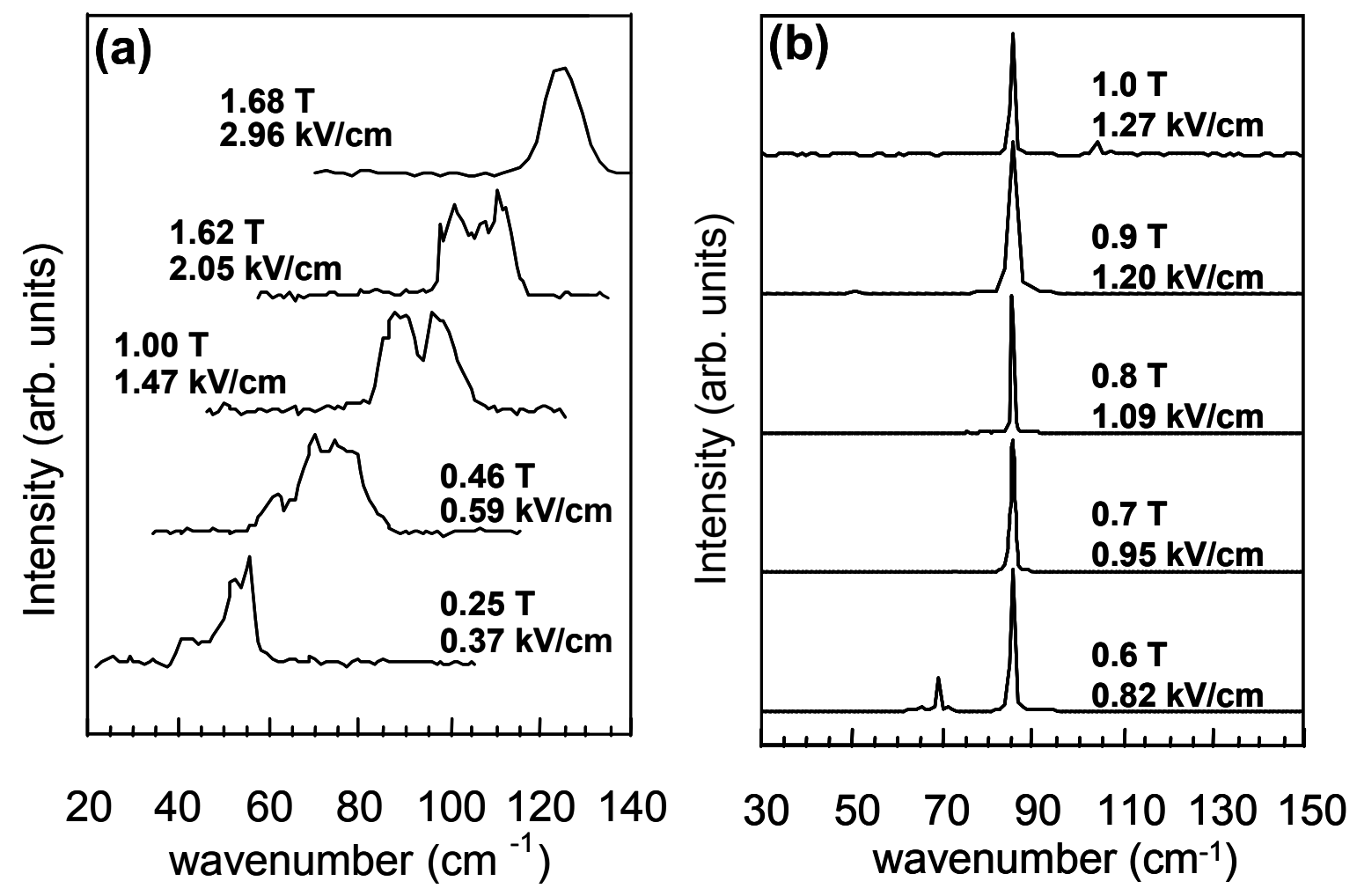

Figure 1, D. R. Chamberlin, E. Bründermann, and E. E. Haller 


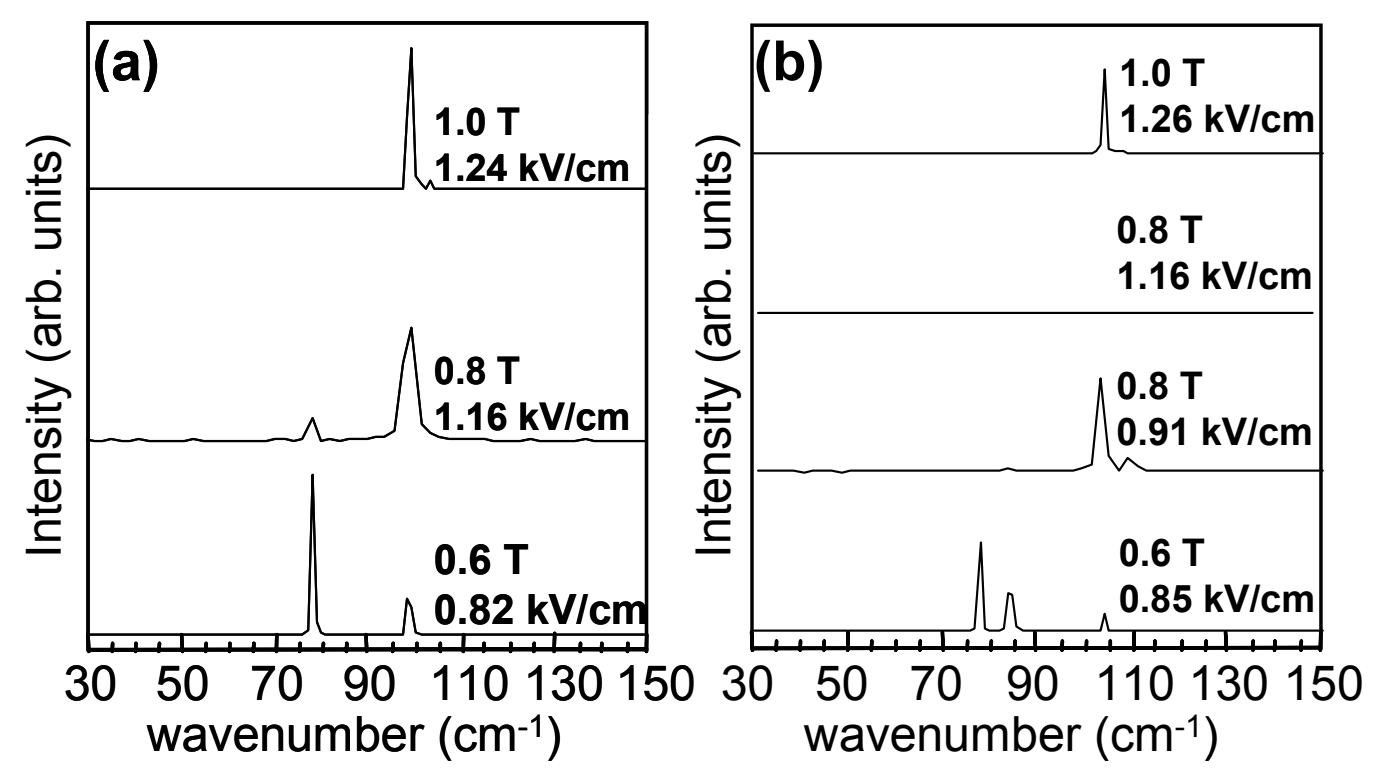

Figure 2, D. R. Chamberlin, E. Bründermann, and E. E. Haller 


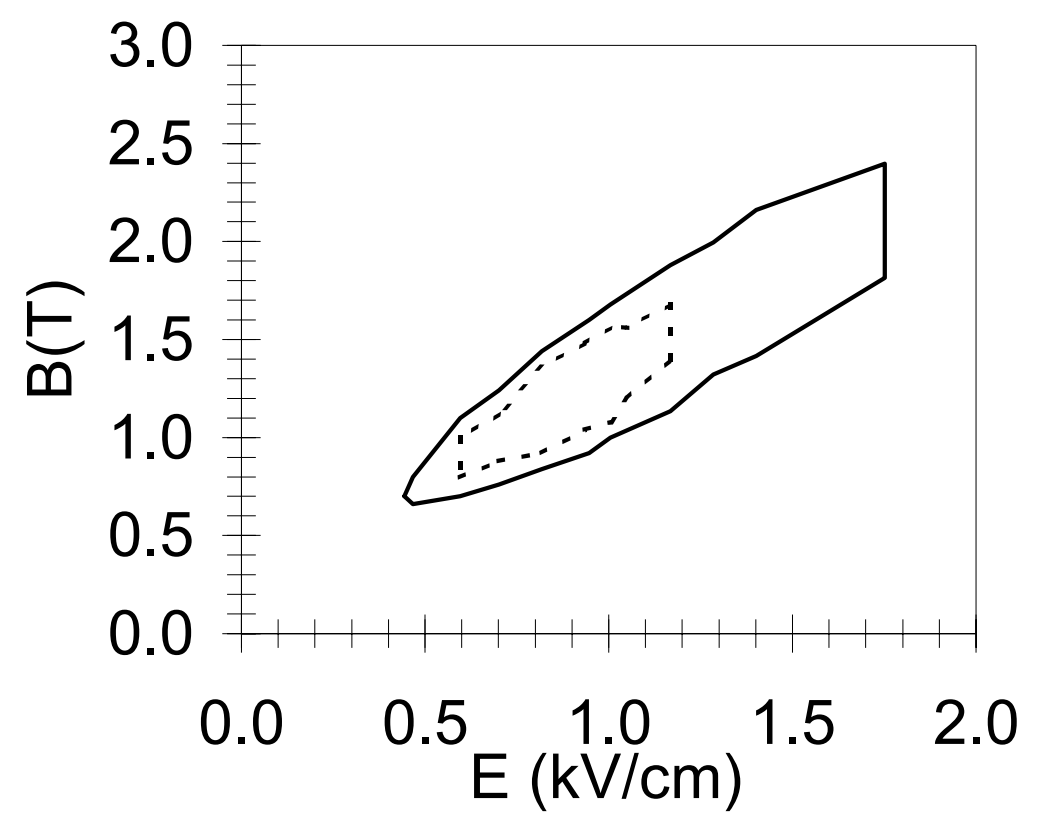

Figure 3: D. R. Chamberlin, E. Bründermann, and E. E. Haller 


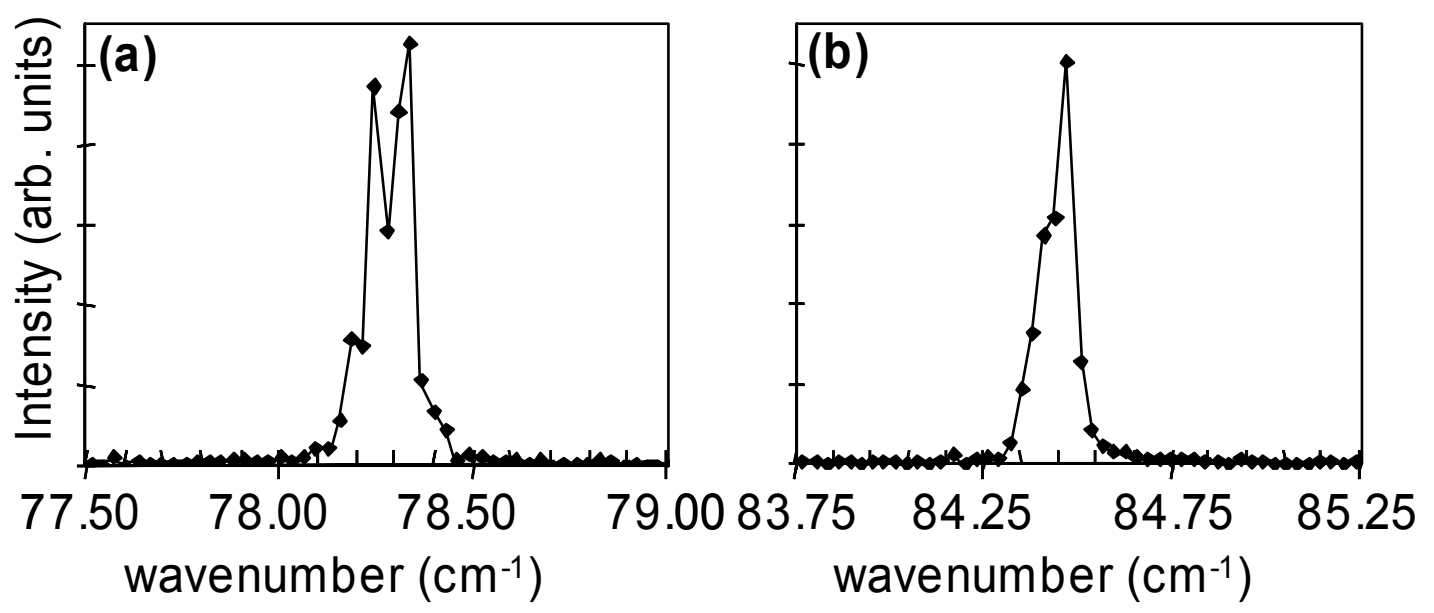

Figure 4: D. R. Chamberlin, E. Bründermann, and E. E. Haller 